\title{
RES with RIB
}

\author{
F. de Oliveira Santos, ${ }^{\text {a }}$ on behalf of E561S and E521S collaborations
}

GANIL, CEA/DSM-CNRS/IN2P3, Caen France.

\begin{abstract}
The Resonant Elastic Scattering (RES) method has been used at GANIL with Radioactive Ions Beams (RIB) in order to study important unbound states. Three examples of studies are given: the first one is related to the important astrophysical reaction ${ }^{18} \mathrm{~F}(\mathrm{p}, \alpha){ }^{15} \mathrm{O}$, the second one to the two-proton radioactivity of ${ }^{19} \mathrm{Mg}$, and the third one to the prediction of the existence of a narrow state in the unbound nucleus ${ }^{15} \mathrm{~F}$. The three experiments have benefited from high quality radioactive beams produced by the Spiral Facility.
\end{abstract}

\section{Introduction}

A century ago, Marsden, Geiger, Rutherford and colleagues measured for the first time the scattering of alpha particles on a thin foil of gold. This famous experiment allowed them to understand the structure of the atom, in particular the presence of a heavy nucleus in the heart of the atom. In this experiment, the incident alpha particles have a very low energy compared to the Coulomb barrier. The differential cross section of the Coulomb scattering can be calculated using the Rutherford formula. It shows that the elastic scattering cross section decreases as $E^{-2}$ when the energy increases. This experiment and its results are widely known, but what is less known is that several targets with different thicknesses were used, not just a thin foil of gold. In experiments that followed this first experience, sometimes the measured cross sections deviated from the values calculated with the Rutherford formula, especially for light nuclei. This might seem mysterious since in these experiments the energy of alpha particles was below the Coulomb barrier.

Figure 1 shows the excitation function of the elastic scattering reaction ${ }^{14} N(p, p){ }^{14} N$ measured recently at GANIL [1]. The Rutherford formula describes very well the overall shape as it is shown with the continuous line, but a resonance is observed at an energy close to $1.06 \mathrm{MeV}$. Obviously this resonance is not explained by a simple Coulomb scattering, while yet the incident energy is well below the Coulomb barrier, here about $2 \mathrm{MeV}$. This resonance is the manifestation of another contribution, it is the fusion of the two incident nuclei forming the compound nucleus ${ }^{15} \mathrm{O}$, which then decays towards the elastic scattering channel. This fusion reaction occurs for two reasons, firstly the penetration of the Coulomb barrier is possible through the quantum tunnel effect, and secondly the existence of a discrete state in the compound nucleus induced a resonant increase in the penetration probability. Thus in general, this kind of measurement can be used to study the compound nucleus. Excitation energies can be determined from resonance po-

\footnotetext{
a e-mail: oliveira@ganil.fr
}

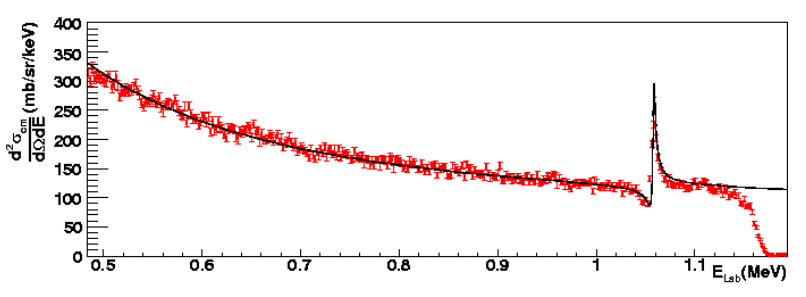

Fig. 1. Measured excitation function of the elastic scattering reaction ${ }^{14} N(p, p){ }^{14} N$ [1]. While the shape of the curve is very well reproduced by the Rutherford formula (continuous line), a peak located at the energy of $1.06 \mathrm{MeV}$ is clearly visible. This peak reflects the existence of a discrete state in the compound nucleus ${ }^{15} \mathrm{O}$.

sitions, the total and partial widths of the states can be extracted from the width and intensity of the resonances, and the spin and parity of the states from the shape of the resonances and the angular distributions. The interest of using such kind of measurement to study the spectroscopy of unbound states is manifest: this measurement is simple, it gives pertinent properties of the states, and the cross sections are often high, these are essential conditions when dealing with RIB [2].

\section{Three examples of RES experiments performed at GANIL}

\subsection{The important astrophysical reaction ${ }^{18} \mathbf{F}(\mathbf{p}, \alpha){ }^{15} \mathbf{O}$}

Stellar explosions called novae take place in stellar binary systems which are made of two stars, a red giant and a small hot companion called a white dwarf. Matter is torn off the red giant and falls onto the surface of the white dwarf. This stellar matter accumulates on the surface of the white dwarf, leading to an increase in its temperature and density. At some density threshold, a runaway of thermonuclear reactions is triggered, producing heavier and 
radioactive elements [3]. The observation of $\gamma$ rays from nova ejecta should provide a rather direct way to investigate the nucleosynthesis and matter ejection mechanism [4]. The most powerful $\gamma$-ray emission coming from novae is predicted to be at energies of $511 \mathrm{keV}$ and below, originating from positron annihilations. It was shown that the main contribution to positron production is the long-lived ${ }^{18} \mathrm{~F}$ radioactive nucleus (half-life $109.77 \mathrm{~min}$ ). Therefore, the amount of radiation emitted scales with the ${ }^{18} \mathrm{~F}$ content of the nova ejecta, which in turn depends strongly on its production and destruction rates. The reaction ${ }^{18} F(p, \alpha){ }^{15} \mathrm{O}$ reaction was identified to be the most sensitive and uncertain reaction for the destruction of ${ }^{18} \mathrm{~F}$ [5]. Despite a lot of experimental efforts [6-8], uncertainties remain in the determination of the rate of this reaction at novae temperatures. One potentially important source of uncertainty comes from interferences between three $\frac{3}{2}^{+}$resonances $[7$, 9]. M. Dufour and P. Descouvemont [10] predicted the existence of two $\frac{1}{2}^{+}$states, one at $0.41 \mathrm{MeV}$ below the proton emission threshold and a second broad resonance at about $1.49 \mathrm{MeV}$ above the threshold. If the existence and properties of these $\frac{1}{2}^{+}$states were confirmed, the reaction rate at typical novae temperatures would be dominated by reactions through these states. It would follow that the importance of the interference contribution between the $\frac{3}{2}^{+}$resonances would be much reduced. Recently, J.C. Dalouzy et al [8] observed for the first time the second resonance using an inelastic scattering reaction and proton-proton correlation technique, but this state was controversial since none of the other experiments could observed it.

A new experiment was proposed at GANIL by A. Murphy (The School of Physics and Astronomy Edinburgh UK) et al in order to study the excited states in ${ }^{19} \mathrm{Ne}$ located above the proton emission threshold, and so to confirm the existence of this new $1 / 2+$ state. The cross sections of the two reactions ${ }^{18} \mathrm{~F}(\mathrm{p}, \alpha){ }^{15} \mathrm{O}$ and ${ }^{18} \mathrm{~F}(\mathrm{p}, \mathrm{p})$ were measured simultaneously in inverse kinematics using a ${ }^{18} \mathrm{~F}$ radioactive beam produced by the Spiral facility and accelerated to 4 $\mathrm{AMeV}$. An important part of the work was performed in order to develop this new beam of ${ }^{18} \mathrm{~F}$ at low energy and with a high purity. It was obtained with an intensity of $2 \times 10^{4} \mathrm{pps}$ and a purity of $97 \%$. A stable beam of ${ }^{18} \mathrm{O}$ was also used in the same experimental conditions for calibrations. A Multi Channel Plate detector was placed upstream the target and was used to measure time of flight and beam intensity. A 6 $\mu \mathrm{m}$ thick foil of gold was used to degrade the energy of the beam down to about $2.3 \mathrm{AMeV}$ and a polyethylene $\left(\mathrm{CH}_{2}\right)_{n}$ target of about $50 \mu \mathrm{m}$ thick was used to induce the reactions. Scattered protons and emitted alpha particles were detected by a Type-W 16x16 DSSD silicon detector placed at forward angles $\left(180^{\circ}\right.$ in the center of mass frame) within an total angular acceptance of about $10^{\circ}$ (lab). Protons and alpha were identified using their energy and time-of-flight. The results of this experiment are still under analysis (Collab.: The School of Physics and Astronomy Edinburgh UK, GANIL, IPN Orsay, University of York UK, ORNL, LPC Caen). An "on-line" spectrum of the $\mathrm{H}\left({ }^{18} \mathrm{~F}, \mathrm{p}\right){ }^{18} \mathrm{~F}$ reaction is shown in Fig. 2, it is compared with several other results obtained in different experiments. The properties of the

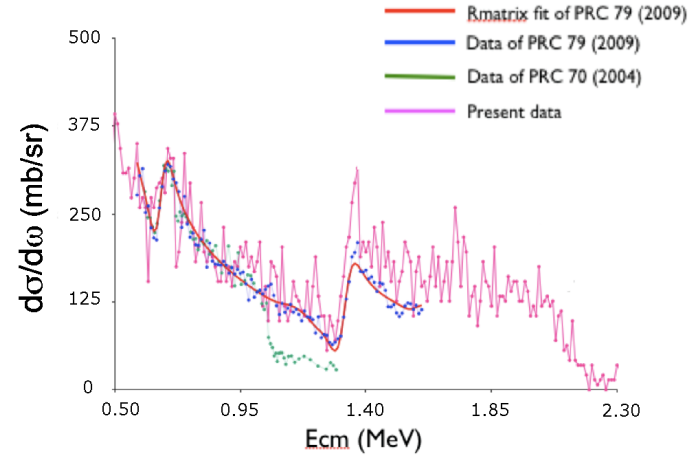

Fig. 2. Preliminary "on-line" results obtained for the excitation function of the $\mathrm{H}\left({ }^{18} \mathrm{~F}, \mathrm{p}\right){ }^{18} \mathrm{~F}$ reaction. This spectrum shows structures that are related to the properties of excited states in ${ }^{19} \mathrm{Ne}$. Our measurement is compared with other results obtained in different experiments

excited states in ${ }^{19} \mathrm{Ne}$ will be obtained using a careful RMatrix analysis of the excitation function constrained with the results obtained for the $\mathrm{H}\left({ }^{18} \mathrm{~F}, \alpha\right){ }^{18} \mathrm{~F}$ reaction. A preliminary analysis has shown that a broad state is required to fit the data [11], which would confirm the existence of this $1 / 2+$ state

\subsection{Two-proton radioactivity of ${ }^{19} \mathrm{Mg}$}

The two-proton radioactivity of ${ }^{19} \mathrm{Mg}$ was discovered recently [12] using a tracking technique, its lifetime was measured to be $4.0(15) \mathrm{ps}$, the $\mathrm{Q}$ value is $0.75(5) \mathrm{MeV}$, and the measured angular correlation between the emitted protons does not show strong di-proton correlation [13]. However, the intermediate unbound nucleus ${ }^{18} \mathrm{Na}$ is almost unknown. It is not yet clear if the ground state of ${ }^{18} \mathrm{Na}$ is located above or below ${ }^{19} \mathrm{Mg}$ ground state $[14,15]$. It is very important to measure the properties of the ${ }^{18} \mathrm{Na}$ low lying states in order to understand the two-proton decay mechanism of ${ }^{19} \mathrm{Mg}$ and the lifetime of this nucleus.

As already discussed, the RES is a powerful method to investigate the structure of unbound nuclei. However very few proton-rich unbound nuclei are accessible experimentally due to very low beam intensities as getting closer to the proton drip-line. The unbound nucleus ${ }^{18} \mathrm{Na}$, the intermediate nucleus in the two-proton radioactivity of ${ }^{19} \mathrm{Mg}$, is one of the rare unbound nucleus which is accessible. We performed the measurement of the RES reaction $\mathrm{p}\left({ }^{17} \mathrm{Ne}, \mathrm{p}\right){ }^{17} \mathrm{Ne}$ in inverse kinematics in order to study these states in ${ }^{18} \mathrm{Na}$. A pure beam of radioactive ${ }^{17} \mathrm{Ne}^{3+}$ ions was produced by the Spiral facility at GANIL with a mean intensity of $10^{4}$ pps and accelerated to $4 \mathrm{~A} . \mathrm{MeV}$. A beam of ${ }^{17} \mathrm{O}^{3+}$ ions was also produced in similar experimental conditions for calibration by comparison with the ${ }^{17} \mathrm{O}\left(\mathrm{p},{ }^{17} \mathrm{O}\right) \mathrm{p}$ reaction measured in direct kinematics. The beam impinged on a fixed $50 \mu \mathrm{m}$ thick polypropylene $\mathrm{C}_{3} \mathrm{H}_{6}$ target coupled to 


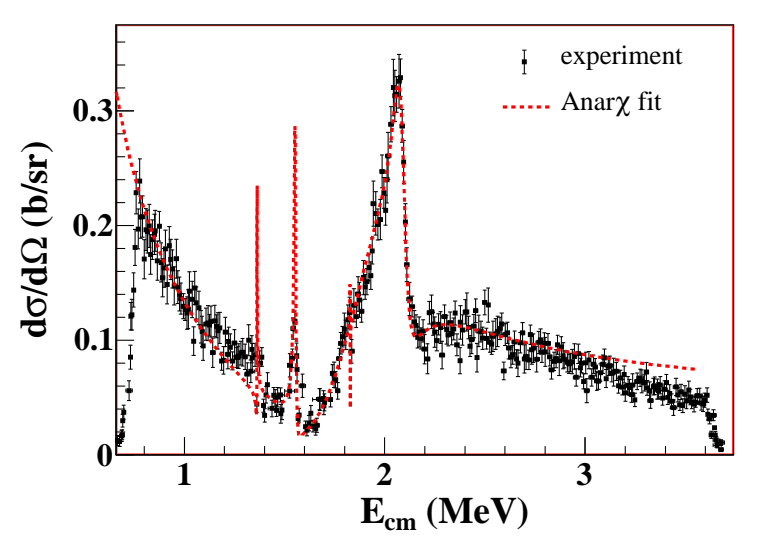

Fig. 3. Measured excitation function of the ${ }^{17} \mathrm{Ne}\left(\mathrm{p},{ }^{17} \mathrm{Ne}\right) \mathrm{p}$ reaction is presented as a function of CM energy. It is measured between 5 and 20 degrees (LAB) and reconstructed at 180 degrees in the CM. Dotted line shows the best fit obtained using the Rmatrix formalism including four low lying states in ${ }^{18} \mathrm{Na}$.

a second rotating $50 \mu \mathrm{m}$ thick $\mathrm{C}_{3} \mathrm{H}_{6}$ target. The two targets together were thick enough to stop the ${ }^{17} \mathrm{Ne}$ beam. This method, described in ref.[2,16], enables us to measure the excitation function at once. Scattered protons were detected by a $\Delta \mathrm{E}-\mathrm{E}$ annular telescope of silicon detectors placed at forward angles. The telescope was composed of a thin $(\approx 40 \mu \mathrm{m})$ double-sided silicon strip detector coupled to a $1.5 \mathrm{~mm}$ thick silicon detector and was covering angles from 5 to 20 degrees in laboratory reference frame. The scattered proton spectrum was polluted by beta-delayed protons emitted in the beta decay of ${ }^{17} \mathrm{Ne}$. This nucleus decays with a lifetime of $0.109 \mathrm{~s}$ and a probability to emit protons of $\sim 90 \%$. More than $98 \%$ of the $\beta$-delayed protons were rejected by using a $60 \mathrm{~cm}$ circular target (FULIS target [17]) rotating at $1000 \mathrm{rpm}$. The ions were implanted in the target and moved away before they decayed. A MCP was used for time of flight and beam measurement with an efficiency close to $100 \%$. From ToF measurement and $\triangle \mathrm{E}-\mathrm{E}$ selection, the scattered protons were identified and the excitation function was reconstructed in the laboratory frame (see Fig. 3). The residual $\beta$-delayed protons were subtracted. The background produced by the presence of ${ }^{12} \mathrm{C}$ in the target was measured using a pure carbon target and was also subtracted. A fit of the excitation function was performed with the R-matrix code Anar $\chi$ [18]. The best fit obtained in this analysis is presented in Fig. 3 (dotted line), it required the presence four low lying states in ${ }^{18} \mathrm{Na}$. The R-matrix calculation agrees very well with the data in most part of the excitation function, including the low energy region where the cross section is mainly described by the Rutherford scattering. This experiment also suggests the additional presence of two very narrow states, including the ground state of ${ }^{18} \mathrm{Na}$. These resonances are too narrow to be seen, this means their width are narrower than $1 \mathrm{keV}$. A detailed description of the experiment and results are presented in [19]. All these measured states in ${ }^{18} \mathrm{Na}$ are located above the ${ }^{19} \mathrm{Mg}$ ground state. The effect

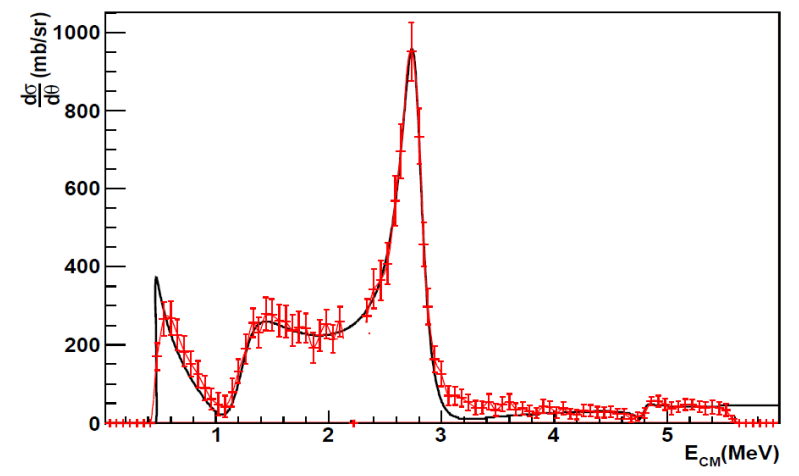

Fig. 4. Measured excitation function of the reaction ${ }^{1} \mathrm{H}\left({ }^{14} \mathrm{O}, \mathrm{p}\right){ }^{14} \mathrm{O}$. At an energy close to $4.8 \mathrm{MeV}$ a hole can be observed, it corresponds to the presence of a narrow excited state in ${ }^{15} \mathrm{~F}$ with a spin $1 / 2-$.

of these results on the two-proton radioactivity mechanism of ${ }^{19} \mathrm{Mg}$ is also discussed in this reference.

\subsection{The existence of a narrow state in ${ }^{15} \mathrm{~F}$}

The unbound nucleus ${ }^{15} \mathrm{~F}$ is located two steps beyond the proton drip-line. The ground state of this nucleus was measured in several experiments, it is very broad with a width close to $1 \mathrm{MeV}$. Recently, Canton et al [20] predicted the existence of a 1/2- narrow excited state in this nucleus, this state would be the second excited state located at an energy close to $5 \mathrm{MeV}$, meaning that it would be located above the Coulomb barrier. In order to confirm the existence of this extraordinary state, we measured the RES reaction ${ }^{1} \mathrm{H}\left({ }^{14} \mathrm{O}, \mathrm{p}\right){ }^{14} \mathrm{O}$ optimizing the experimental conditions in order to obtain the best energy resolution. The experiment was performed at GANIL using an ${ }^{14} \mathrm{O}$ RIB produced by the SPIRAL facility and post accelerated to $6 \mathrm{MeV} / \mathrm{u}$ with the CIME cyclotron. After using a stripper foil, the beam purity was close to $100 \%$ and the mean intensity was $2 \times 10^{5} \mathrm{pps}$. The beam impinged on a $150 \mu \mathrm{m}$ thick rotating polypropylene target (the same system as in the ${ }^{18} \mathrm{Na}$ experiment). Scattered protons were detected with a $\Delta \mathrm{E}(500 \mu \mathrm{m})-\mathrm{E}(6 \mathrm{~mm} \mathrm{SiLi})$ telescope of silicon detectors located $36.6 \mathrm{~cm}$ downstream the target. The angular acceptance was \pm 2.16 degrees in laboratory and the energy resolution was $50 \mathrm{keV}$. The measured excitation function is shown in figure 4 . The first large hole that can be observed at the energy close to $1 \mathrm{MeV}$ corresponds to the ground state of ${ }^{15} \mathrm{~F}$ which has a spin $1 / 2+$. The peak at the energy close to $2.8 \mathrm{MeV}$ corresponds to the known $5 / 2+$ first excited state. At an energy close to $4.8 \mathrm{MeV}$ a small reduction of the crosse section can be observed in agreement with the presence of a narrow 1/2- state, as it was predicted in [20]. The width of the state is about $50 \mathrm{keV}$. The fact that this state has a width very small relatively to the ground state width may seem surprising. This is even more surprising because this state is located above the Coulomb barrier, no barrier therefore hold back the proton leaving the nucleus and the width could have been larger than $1 \mathrm{MeV}$. In 
fact, this state is mainly described as a configuration ${ }^{13} \mathrm{~N}$ $+2 \mathrm{p}$ (spectroscopic factor close to 1 ), and not as a configuration ${ }^{14} \mathrm{O}+\mathrm{p}$. This explains why the proton width is so narrow. Moreover, the two-protons decay is greatly reduced because the energy decay is very small, about 150 $\mathrm{keV}$ [21].

\section{References}

1. I. Stefan, Thesis (Université de Caen France,2007).

2. F. de Oliveira Santos et al., Eur. Phys. J. A 24, (2005) 237

3. J. José and M. Hernanz and C. Iliadis, Nucl. Phys. A 777, (2006) 550-578

4. J. Gomez-Gomar et al., MNRAS 296, (1998) 913

5. A. Coc and M. Hernanz and J. José and J.-P. Thibaud, Astron. Astrophys. 357, (2000) 561

6. S. Utku et al., Phys. Rev. C 57, (1998) 2731

7. N. de Séréville et al., Nuclei in the Cosmos-IX (2006) 05

8. J.C. Dalouzy et al., Phys. Rev. Lett. 102, (2009) 16

9. C. D. Nesaraja et al., Phys. Rev. C 75, (2007) 055809

10. M. Dufour and P. Descouvemont, Nucl. Phys. A 785, (2007) 785

11. D. Mountford et al., to be submitted. D. Mountford thesis. University of Edinburgh.

12. I.G. Mukha et al, Phys. Rev. Lett., 99 (2007) 182501.

13. I. Mukha et al, Phys. Rev. C, 77 (2008) 061303(R).

14. T. Zerguerras et al, Eur. Phys. J. A, 20 (2004) 389.

15. H. T. Fortune and R. Sherr, Phys. Rev. C, 72 (2005) 034304.

16. V.Z. Goldberg et al, Phys. At. Nucl. 60 (1997) 1186.

17. Ch. Stodel et al, Proceeding World Scientific EXON, Peterhof, Russia, July 2004, 180-187.

18. E. Berthoumieux, B. Berthier, C. Moreau, J.P. Gallien and A.C. Raoux, Nucl. Instr. and Meth. B, 136-138 (1998) 55.

19. M. Assié et al, to be submitted.

20. L. Canton et al, Phys. Rev. Lett. 96, (2006) 72502.

21. F. de Grancey et al, to be submitted and Thesis (Université de Caen France,2007). 